\title{
Quantization of a Friedmann-Robertson-Walker model in N=1 Supergravity with Gauged Supermatter
}

\author{
A.D.Y. Cheng, P.D. D'Eath and P.R.L.V. Moniz* \\ Department of Applied Mathematics and Theoretical Physics \\ University of Cambridge, Silver Street,Cambridge CB3 9EW, UK
}

\begin{abstract}
The theory of $\mathrm{N}=1$ supergravity with gauged supermatter is studied in the context of a $\mathrm{k}=+1$ Friedmann minisuperspace model. It is found by imposing the Lorentz and supersymmetry constraints that there are no physical states in the particular $S U(2)$ model studied.

The subjects of supersymmetric quantum gravity and cosmology have achieved a considerable number of very interesting results and conclusions during the last ten years or so $[2,3]$. Our objective here is to study a $k=1$ supersymmetric FRW mini-superspace quantum cosmological model with a family of spin-0 as well as spin-1 gauge fields together with their odd (anti-commuting) spin- $\frac{1}{2}$ partners with zero analytic potential $P\left(\Phi^{I}\right)$. The supersymmetry constraints will be derived from the reduced theory with supermatter. Subsequently, we solve for the components of the wave function using the quantum constraints. We will then find that there are no solutions for the quantum states of the FRW universe analysed here.

Let us begin by specifying our model in some detail. The Lagrangian of the theory studied here is given in Eq. (25.12) of ref. [1]; it is too long to write out here. We choose the geometry to be that of a $k=+1$ Friedmann model with $S^{3}$ spatial sections, which are the spatial orbits of $G=S O(4)$ - the group of homogeneity and isotropy. The tetrad of the four-dimensional theory can be taken to be:

$$
e_{a \mu}=\left(\begin{array}{cc}
N(\tau) & 0 \\
0 & a E_{\hat{a} i}
\end{array}\right), \quad e^{a \mu}=\left(\begin{array}{cc}
N(\tau)^{-1} & 0 \\
0 & a(\tau)^{-1} E^{\hat{a} i}
\end{array}\right)
$$
\end{abstract}

where $\hat{a}$ and $i$ run from 1 to 3 . $E_{\hat{a} i}$ is a basis of left-invariant 1 -forms on the unit $S^{3}$ with volume $\sigma^{2}=2 \pi^{2}$. The spatial tetrad $e^{A A^{\prime}}{ }_{i}$ satisfies the relation

$$
\partial_{i} e^{A A^{\prime}}{ }_{j}-\partial_{j} e^{A A^{\prime}}=2 a^{2} e_{i j k} e^{A A^{\prime} k}
$$

as a consequence of the group structure of $\mathrm{SO}(3)$, the isotropy (sub)group.

This Ansatz reduces the number of degrees of freedom provided by $e_{A A^{\prime} \mu}$. If supersymmetry invariance is to be retained, then we need an Ansatz for $\psi_{\mu}^{A}$ and $\bar{\psi}_{\mu}^{A^{\prime}}$ which

* e-mail address: prlvm10@amtp.cam.ac.uk 
reduces the number of fermionic degrees of freedom, so that there is equality between the number of bosonic and fermionic degrees of freedom. One is naturally led to take $\psi_{0}^{A}$ and $\bar{\psi}^{A^{\prime}}$ to be functions of time only. We further take

$$
\psi_{i}^{A}=e^{A A^{\prime}} \bar{\psi}_{A^{\prime}}, \bar{\psi}_{i}^{A^{\prime}}=e^{A A^{\prime}}{ }_{i} \psi_{A}
$$

where we introduce the new spinors $\psi_{A}$ and $\bar{\psi}_{A^{\prime}}$ which are functions of time only. [It is possible to justify the above Ansatz by requiring that the form of the tetrad be preserved under suitable homogeneous supersymmetry transformations. [2]

Now, consider the supermatter fields. The scalar super-multiplet, consisting of a complex massive scalar field $\phi$ and massive spin- $\frac{1}{2}$ field $\chi, \bar{\chi}$ are chosen to be spatially homogeneous, depending only on time. The odd spin- $\frac{1}{2} \operatorname{partner}\left(\lambda^{(a)}, \bar{\lambda}^{(a)}\right), a=1,2,3$, is chosen to depend only on time as well. As far as the vector field $A_{\mu}^{(a)}$ is concerned we adopt here the Ansatz formulated in ref. [4] and choose

$$
\mathbf{A}_{\mu}(t) \omega^{\mu}=\frac{f(t)}{2} \mathcal{T}_{c} \omega^{c}
$$

where $\left\{\omega^{\mu}\right\}$ represents the moving coframe $\left\{\omega^{\mu}\right\}=\left\{d t, \omega^{b}\right\},(b=1,2,3)$, of one-forms, invariant under the left action of $S U(2)$ and $\mathcal{T}_{a}$ are the generators of the $S U(2)$ gauge group. Notice in the above form for the gauge field the $A_{0}$ component is taken to be identically zero. Thus, we will have in our FRW case a gauge constraint $Q^{(a)}=0$.

Using the Ansätze previously described, the action of the full theory (Eq. (25.12) in ref. [1]) can be reduced to one with a finite number of degrees of freedom. Notice that with our choice of gauge group $S U(2)$ and compact Kähler manifold, itdirectly follows that the analytical potential $P\left(\Phi^{I}\right)$ is zero [5]

Let us here solve explicitly the corresponding quantum supersymmetry constraints. First we need to redefine the fermionic fields, $\chi_{A}, \psi_{A}$ and $\lambda_{A}$ in order to simplify the Dirac brackets, following the steps described in ref. [6]:

$$
\begin{gathered}
\hat{\chi}_{A}=\frac{\sigma a^{\frac{3}{2}}}{2^{\frac{1}{4}}(1+\phi \bar{\phi})} \chi_{A}, \hat{\bar{\chi}}_{A^{\prime}}=\frac{\sigma a^{\frac{3}{2}}}{2^{\frac{1}{4}}(1+\phi \bar{\phi})} \bar{\chi}_{A^{\prime}}, \pi_{\hat{\chi}_{A}}=-i n_{A A^{\prime}} \hat{\bar{\chi}}^{A^{\prime}}, \pi_{\hat{\bar{\chi}}_{A^{\prime}}}=-i n_{A A^{\prime}} \hat{\chi}^{A} \\
\hat{\psi}_{A}=\frac{\sqrt{3}}{2^{\frac{1}{4}}} \sigma a^{\frac{3}{2}} \psi_{A}, \hat{\bar{\psi}}_{A^{\prime}}=\frac{\sqrt{3}}{2^{\frac{1}{4}}} \sigma a^{\frac{3}{2}} \bar{\psi}_{A^{\prime}}, \pi_{\hat{\psi}_{A}}=i n_{A A^{\prime}} \hat{\bar{\psi}}^{A^{\prime}}, \pi_{\hat{\bar{\psi}}_{A^{\prime}}}=i n_{A A^{\prime}} \hat{\psi}^{A} \\
\hat{\lambda}_{A}^{(a)}=\frac{\sigma a^{\frac{3}{2}}}{2^{\frac{1}{4}}} \lambda_{A}^{(a)}, \quad \hat{\bar{\lambda}}_{A^{\prime}}^{(a)}=\frac{\sigma a^{\frac{3}{2}}}{2^{\frac{1}{4}}} \bar{\lambda}_{A^{\prime}}^{(a)}, \pi_{\hat{\lambda}_{A}^{(a)}}=-i n_{A A^{\prime}} \hat{\bar{\lambda}}^{(a) A^{\prime}}, \pi_{\hat{\bar{\lambda}}_{A^{\prime}}^{(a)}}=-i n_{A A^{\prime}} \hat{\lambda}^{(a) A}
\end{gathered}
$$


The Dirac brackets are:

$$
\begin{gathered}
{\left[\hat{\chi}_{A}, \hat{\bar{\chi}}_{A^{\prime}}\right]_{*}=-i n_{A A^{\prime}},\left[\hat{\psi}_{A}, \hat{\bar{\psi}}_{A^{\prime}}\right]_{*}=i n_{A A^{\prime}},\left[\hat{\lambda}_{A}^{(a)}, \hat{\bar{\lambda}}_{A^{\prime}}^{(a)}\right]_{*}=-i \delta^{a b} n_{A A^{\prime}}} \\
{\left[a, \pi_{a}\right]_{*}=1,\left[\phi, \pi_{\phi}\right]_{*}=1,\left[\bar{\phi}, \pi_{\bar{\phi}}\right]_{*}=1,\left[f, \pi_{f}\right]=1}
\end{gathered}
$$

and the rest of the brackets are zero. It is simpler to describe the theory using only (say) unprimed spinors, and, to this end, we define

$$
\bar{\psi}_{A}=2 n_{A}^{B^{\prime}} \bar{\psi}_{B^{\prime}}, \bar{\chi}_{A}=2 n_{A}^{B^{\prime}} \bar{\chi}_{B^{\prime}}, \bar{\lambda}_{A}^{(a)}=2 n_{A}^{B^{\prime}} \bar{\lambda}_{B^{\prime}}^{(a)}
$$

with which the new Dirac brackets are

$$
\left[\chi_{A}, \bar{\chi}_{B}\right]_{*}=-i \epsilon_{A B},\left[\psi_{A}, \bar{\psi}_{B}\right]_{*}=i \epsilon_{A B},\left[\lambda_{A}^{(a)}, \bar{\lambda}_{A^{\prime}}^{(a)}\right]_{*}=-i \delta^{a b} \epsilon_{A B}
$$

The rest of the brackets remain unchanged. Hence the only non-zero (anti-)commutator relations are:

$$
\begin{gathered}
\left\{\lambda_{A}^{(a)}, \lambda_{B}^{(b)}\right\}=\delta^{a b} \epsilon_{A B},\left\{\chi_{A}, \bar{\chi}_{B}\right\}=\epsilon_{A B},\left\{\psi_{A}, \bar{\psi}_{B}\right\}=-\epsilon_{A B} \\
{\left[a, \pi_{a}\right]=\left[\phi, \pi_{\phi}\right]=\left[\bar{\phi}, \pi_{\bar{\phi}}\right]=\left[f, \pi_{f}\right]=i}
\end{gathered}
$$

Here we choose $\left(\chi_{A}, \psi_{A}, a, \phi, \bar{\phi}\right)$ to be the coordinates of the configuration space, and $\bar{\chi}_{A}, \bar{\psi}_{A}, \pi_{a}, \pi_{\phi}, \pi_{\bar{\phi}}$ to be the momentum operators in this representation. Hence

$$
\begin{gathered}
\lambda_{A}^{a} \rightarrow-\frac{\partial}{\partial \bar{\lambda}^{(a) A}}, \bar{\chi}^{A} \rightarrow-\frac{\partial}{\partial \chi^{A}}, \bar{\psi}_{A} \rightarrow \frac{\partial}{\partial \psi^{A}} \\
\pi_{a} \rightarrow \frac{\partial}{\partial a}, \pi_{\phi} \rightarrow-i \frac{\partial}{\partial \phi}, \pi_{\bar{\phi}} \rightarrow-i \frac{\partial}{\partial \phi}, \pi_{f} \rightarrow-i \frac{\partial}{\partial f}
\end{gathered}
$$

Following the ordering used in ref. [2], we put all the fermionic derivatives in $S_{A}$ on the right. In $\bar{S}_{A}$, all the fermonic derivatives are on the left. Implementing all these redefinitions, the supersymmetry constraints have the differential operator form

$$
\begin{aligned}
& S_{A}=-\frac{i}{\sqrt{2}}(1+\phi \bar{\phi}) \chi_{A} \frac{\partial}{\partial \phi}-\frac{1}{2 \sqrt{6}} a \psi_{A} \frac{\partial}{\partial a}-\sqrt{\frac{3}{2}} \sigma^{2} a^{2} \psi_{A}-\frac{5 i}{4 \sqrt{2}} \bar{\phi} \chi_{A} \chi^{B} \frac{\partial}{\partial \chi^{B}} \\
& -\frac{1}{8 \sqrt{6}} \psi_{B} \psi^{B} \frac{\partial}{\partial \psi^{A}}-\frac{i}{4 \sqrt{2}} \bar{\phi} \chi_{A} \psi^{B} \frac{\partial}{\partial \psi^{B}}-\frac{5}{4 \sqrt{6}} \chi_{A} \psi^{B} \frac{\partial}{\partial \chi^{B}}+\frac{\sqrt{3}}{4 \sqrt{2}} \chi^{B} \psi_{B} \frac{\partial}{\partial \chi^{A}}
\end{aligned}
$$




$$
\begin{gathered}
+\frac{1}{2 \sqrt{6}} \psi_{A} \chi^{B} \frac{\partial}{\partial \chi^{B}}+\frac{1}{3 \sqrt{6}} \sigma_{A B^{\prime}}^{a} \sigma^{b C C^{\prime}} n_{D}^{B^{\prime}} n_{C^{\prime}}^{B} \bar{\lambda}^{(a) D} \psi_{C} \frac{\partial}{\partial \bar{\lambda}^{(b) B}} \\
+\frac{1}{6 \sqrt{6}} \sigma_{A B^{\prime}}^{a} \sigma^{b B A^{\prime}} n_{D}^{B^{\prime}} n_{A^{\prime}}^{E} \bar{\lambda}^{(a) D} \bar{\lambda}^{(b)}{ }_{B} \frac{\partial}{\partial \psi^{E}}-\frac{1}{2 \sqrt{6}} \psi_{A} \bar{\lambda}^{(a) C} \frac{\partial}{\bar{\lambda}^{(a) C}}+\frac{3}{8 \sqrt{6}} \bar{\lambda}^{a}{ }_{A} \lambda^{(a) C} \frac{\partial}{\partial \psi^{C}} \\
+\frac{1}{2 \sqrt{2}} \sigma^{2} a^{3} g \bar{D}^{(a)} \bar{\lambda}_{A}^{a}-\frac{1}{4 \sqrt{6}} \psi^{C} \bar{\lambda}_{C}^{(a)} \frac{\partial}{\partial \bar{\lambda}^{(a) A}}+\frac{\sigma^{2} a^{2} g f}{\sqrt{2}(1+\phi \bar{\phi})} \sigma_{A A^{\prime}} n^{B A^{\prime}} \bar{X}^{(a)} \chi_{B} \\
+\sigma_{A A^{\prime}}^{a} n^{B A^{\prime}} \bar{\lambda}_{B}^{(a)}\left(-\frac{\sqrt{2}}{3} \frac{\partial}{\partial f}+\frac{1}{8 \sqrt{2}}\left(1-(f-1)^{2}\right) \sigma^{2} a^{4}\right)
\end{gathered}
$$

We now proceed to find the wavefunction of our model. The Lorentz constraint $J_{A B}$ is easy to solve. It tells us that the wave function should be a Lorentz scalar. We can see that the most general form of the wave function which satisfies the Lorentz constraint is

$$
\begin{aligned}
& \Psi=A+i B \psi^{C} \psi_{C}+C \psi^{C} \chi_{C}+i D \chi^{C} \chi_{C}+E \psi^{C} \psi_{C} \chi^{C} \chi_{C} \\
& +c_{a} \bar{\lambda}^{(a) C} \chi_{C}+d_{a} \bar{\lambda}^{(a) C} \psi_{C}+c_{a b} \bar{\lambda}^{(a) C} \bar{\lambda}_{C}^{(b)}+e_{a} \bar{\lambda}^{(a) C} \chi_{C} \psi^{D} \psi_{D} \\
& +f_{a} \bar{\lambda}^{(a) C} \psi_{C} \chi^{D} \chi_{D}+d_{a b} \bar{\lambda}^{(a) C} \chi_{C} \bar{\lambda}^{(a) D} \chi_{D}+e_{a b} \bar{\lambda}^{(a) C} \bar{\lambda}_{C}^{(b)} \psi^{D} \psi_{D} \\
& +f_{a b} \bar{\lambda}^{(a) C} \bar{\lambda}_{C}^{(b)} \chi^{D} \chi_{D}+g_{a b} \bar{\lambda}^{(a) C} \bar{\lambda}_{C}^{(b)} \chi^{D} \psi_{D}+c_{a b c} \bar{\lambda}^{(a) C} \bar{\lambda}_{C}^{(b)} \bar{\lambda}^{(c) D} \psi_{D} \\
& +d_{a b c} \bar{\lambda}^{(a) C} \bar{\lambda}_{C}^{(b)} \bar{\lambda}^{(c) D} \chi_{D}+c_{a b c d} \bar{\lambda}^{(a) C} \bar{\lambda}_{C}^{(b)} \bar{\lambda}^{(c) D} \bar{\lambda}_{D}^{(d)}+h_{a b} \bar{\lambda}^{(a) C} \bar{\lambda}_{C}^{(b)} \psi^{D} \psi_{D} \chi^{E} \chi_{E} \\
& +e_{a b c} \bar{\lambda}^{(a) C} \bar{\lambda}_{C}^{(b)} \bar{\lambda}^{(c) D} \chi_{D} \psi^{E} \psi_{E}+f_{a b c} \bar{\lambda}^{(a) C} \bar{\lambda}_{C}^{(b)} \bar{\lambda}^{(c) D} \psi_{D} \chi^{E} \chi_{E}+d_{a b c d} \bar{\lambda}^{(a) C} \bar{\lambda}_{C}^{(b)} \bar{\lambda}^{(c) D} \bar{\lambda}_{D}^{(d)} \psi^{E} \psi_{E} \\
& +e_{a b c d} \bar{\lambda}^{(a) C} \bar{\lambda}_{C}^{(b)} \bar{\lambda}^{(c) D} \bar{\lambda}_{D}^{(d)} \chi^{E} \chi_{E}+f_{a b c d} \bar{\lambda}^{(a) C} \bar{\lambda}_{C}^{(b)} \bar{\lambda}^{(c) D} \bar{\lambda}_{D}^{(d)} \psi^{E} \chi_{E}+g_{a b c d} \bar{\lambda}^{(a) C} \bar{\lambda}_{C}^{(b)} \bar{\lambda}^{(c) D} \psi_{D} \bar{\lambda}^{(d) E} \chi_{E} \\
& \mu_{1} \bar{\lambda}^{(2) C} \bar{\lambda}_{C}^{(2)} \bar{\lambda}^{(3) D} \bar{\lambda}_{D}^{(3)} \bar{\lambda}^{(1) E} \psi_{E}+\mu_{2} \bar{\lambda}^{(1) C} \bar{\lambda}_{C}^{(1)} \bar{\lambda}^{(3) D} \bar{\lambda}_{D}^{(3)} \bar{\lambda}^{(2) E} \psi_{E}+\mu_{3} \bar{\lambda}^{(1) C} \bar{\lambda}_{C}^{(1)} \bar{\lambda}^{(2) D} \bar{\lambda}_{D}^{(2)} \bar{\lambda}^{(3) E} \psi_{E} \\
& +\nu_{1} \bar{\lambda}^{(2) C} \bar{\lambda}_{C}^{(2)} \bar{\lambda}^{(3) D} \bar{\lambda}_{D}^{(3)} \bar{\lambda}^{(1) E} \chi_{E}+\nu_{2} \bar{\lambda}^{(1) C} \bar{\lambda}_{C}^{(1)} \bar{\lambda}^{(3) D} \bar{\lambda}_{D}^{(3)} \bar{\lambda}^{(2) E} \chi_{E}+\nu_{3} \bar{\lambda}^{(1) C} \bar{\lambda}_{C}^{(1)} \bar{\lambda}^{(2) D} \bar{\lambda}_{D}^{(2)} \bar{\lambda}^{(3) E} \chi_{E} \\
& +F \bar{\lambda}^{(1) C} \bar{\lambda}_{C}^{(1)} \bar{\lambda}^{(2) D} \bar{\lambda}_{D}^{(2)} \bar{\lambda}^{(3) E} \bar{\lambda}_{E}^{(3)}+h_{a b c d} \bar{\lambda}^{(a) C} \bar{\lambda}_{C}^{(b)} \bar{\lambda}^{(c) D} \bar{\lambda}_{D}^{(d)} \psi^{E} \psi_{E} \chi^{F} \chi_{F} \\
& +\delta_{1} \bar{\lambda}^{(2) C} \bar{\lambda}_{C}^{(2)} \bar{\lambda}^{(3) D} \bar{\lambda}_{D}^{(3)} \bar{\lambda}^{(1) E} \psi_{E} \chi^{F} \chi_{F}+\delta_{2} \bar{\lambda}^{(1) C} \bar{\lambda}_{C}^{(1)} \bar{\lambda}^{(3) D} \bar{\lambda}_{D}^{(3)} \bar{\lambda}^{(2) E} \psi_{E} \chi^{F} \chi_{F} \\
& +\delta_{3} \bar{\lambda}^{(1) C} \bar{\lambda}_{C}^{(1)} \bar{\lambda}^{(2) D} \bar{\lambda}_{D}^{(2)} \bar{\lambda}^{(3) E} \psi_{E} \chi^{F} \chi_{F}+\gamma_{1} \bar{\lambda}^{(2) C} \bar{\lambda}_{C}^{(2)} \bar{\lambda}^{(3) D} \bar{\lambda}_{D}^{(3)} \bar{\lambda}^{(1) E} \chi_{E} \psi^{F} \psi_{F} \\
& +\gamma_{2} \bar{\lambda}^{(1) C} \bar{\lambda}_{C}^{(1)} \bar{\lambda}^{(3) D} \bar{\lambda}_{D}^{(3)} \bar{\lambda}^{(2) E} \chi_{E} \psi^{F} \psi_{F}+\gamma_{3} \bar{\lambda}^{(1) C} \bar{\lambda}_{C}^{(1)} \bar{\lambda}^{(2) D} \bar{\lambda}_{D}^{(2)} \bar{\lambda}^{(3) E} \chi_{E} \psi^{F} \psi_{F} \\
& +G \bar{\lambda}^{(1) C} \bar{\lambda}_{C}^{(1)} \bar{\lambda}^{(2) D} \bar{\lambda}_{D}^{(2)} \bar{\lambda}^{(3) E} \bar{\lambda}_{E}^{(3)} \psi^{F} \psi_{F}+H \bar{\lambda}^{(1) C} \bar{\lambda}_{C}^{(1)} \bar{\lambda}^{(2) D} \bar{\lambda}_{D}^{(2)} \bar{\lambda}^{(3) E} \bar{\lambda}_{E}^{(3)} \chi^{F} \chi_{F}
\end{aligned}
$$




$$
+I \bar{\lambda}^{(1) C} \bar{\lambda}_{C}^{(1)} \bar{\lambda}^{(2) D} \bar{\lambda}_{D}^{(2)} \bar{\lambda}^{(3) E} \bar{\lambda}_{E}^{(3)} \chi^{F} \psi_{F}+K \bar{\lambda}^{(1) C} \bar{\lambda}_{C}^{(1)} \bar{\lambda}^{(2) D} \bar{\lambda}_{D}^{(2)} \bar{\lambda}^{(3) E} \bar{\lambda}_{E}^{(3)} \psi^{F} \psi_{F} \chi^{G} \chi_{G}
$$

where $A, B, C, D, E$ etc are functions of $a, \phi$ and $\bar{\phi}$ only. This Ansatz contains all allowed combinations of the fermionic fields and is the most general Lorentz invariant function.

The next step is to solve the supersymmetry constraints $S_{A} \Psi=0$ and $\bar{S}_{A^{\prime}} \Psi=0$. Since each order in fermionic variables is independent, the number of constraint equations will be very high. Their full analysis is quite tedious and to write all the terms would overburden the reader. Let us show some examples of the calculations involved in solving the $S_{A} \Psi=0$ constraint.

Consider the terms linear in $\chi_{A}$ :

$$
\left[-\frac{i}{\sqrt{2}}(1+\phi \bar{\phi}) \frac{\partial A}{\partial \phi}\right] \chi_{A}+\frac{\sigma^{2} a^{2} g f}{\sqrt{2}(1+\phi \bar{\phi})} \sigma_{A A^{\prime}}^{a} n^{B A^{\prime}} \bar{X}^{(a)} A \chi_{B}=0
$$

Since this is true for all $\chi_{A}$, the above equation becomes

$$
\left[-\frac{i}{\sqrt{2}}(1+\phi \bar{\phi}) \frac{\partial A}{\partial \phi}\right] \epsilon_{A}^{B}+\frac{\sigma^{2} a^{2} g f}{\sqrt{2}(1+\phi \bar{\phi})} \sigma_{A A^{\prime}} n^{B A^{\prime}} \bar{X}^{(a)} A=0 .
$$

Mutliplying the whole equation by $n_{B B^{\prime}}$ and using the relation $n_{B B^{\prime}} n^{B A^{\prime}}=\frac{1}{2} \epsilon_{B^{\prime}}^{A^{\prime}}$, we can see that the two terms are independent of each other since the $\sigma$ matrices are orthogonal to the $\mathrm{n}$ matrix. Thus, we conclude that $A=0$. As we proceed, this pattern keeps repeating itself. Some equations show that the coefficients have some symmetry properties. For example, $d_{a b}=2 g_{a b}$. But when these two terms are combined with each other, they become zero. It can be seen as follows,

$$
\begin{aligned}
& d_{a b} \bar{\lambda}^{(a) C} \chi_{C} \bar{\lambda}^{(a) D} \psi_{D}+g_{a b} \bar{\lambda}^{(a) C} \bar{\lambda}_{C}^{(b)} \chi^{D} \psi_{D} \\
= & 2 g_{a b} \bar{\lambda}^{(a) C} \bar{\lambda}^{(b) D} \chi_{D} \psi_{C}+g_{a b} \bar{\lambda}^{(a) C} \bar{\lambda}_{C}^{(b)} \chi^{D} \psi_{D} \\
= & -g_{a b} \bar{\lambda}^{(a) C} \bar{\lambda}_{C}^{(b)} \chi^{D} \psi_{D}+g_{a b} \bar{\lambda}^{(a) C} \bar{\lambda}_{C}^{(b)} \chi^{D} \psi_{D}
\end{aligned}
$$

using the property that $g_{a b}=g_{b a}$ and the spinor identity $\theta_{A B}=\frac{1}{2} \theta_{C}^{C} \epsilon_{A B}$ where $\theta_{A B}$ is antisymetric in the two indices. The same property applies to the terms with coefficients $f_{a b c d}$ and $g_{a b c d}$. Other equations imply that the coefficients $c_{a b c}, d_{a b c}, c_{a b c d}, e_{a b c}, f_{a b c}, d_{a b c d}$, $e_{a b c d}, h_{a b c d}$ are totally symmetric in their indices. This then leads to the terms cancelling with each other, as can easily be shown. In the end, considering both the $S_{A} \Psi=0$ and $\bar{S}_{A} \Psi=0$ constraints, we are left with the surprising result that the wave function must be zero in order to satisfy the quantum constraints. 
To summarise, we have applied the canonical formulation of the more general theory of $N=1$ supergravity with supermatter [6] to a $k=+1$ FRW mini-superspace model, subject to suitable Ansätze for the the gravitational field, gravitino field and the gauge vector field $A_{\mu}^{a}$ as well as the scalar fields and corresponding fermionic partners. After a dimensional reduction, we derived the supersymmetric constraints for our one-dimensional model. We then solved the Lorentz and supersymmetry constraints for the case of a twodimensional spherically symmetric Kähler manifold. We found that there are no physical states in this model. A similar conclusion was also obtained in ref. $[7,8]$ where no matter but a cosmological constant term was present. All this seems to suggest that as one introduces more terms in a locally supersymmetric action,giving more field modes with associated mixing, then the constraints impose severe restrictions on the possible allowed states, assuming homogeneity and isotropy. This is not to say that there might not be many inhomogenous states.

\section{ACKNOWLEDGEMENTS}

The authors are grateful to S.W. Hawking for helpful comments and to J. Mourão for useful conversations. Discussions with R. Capovilla and O. Óbregon are also acknowledged. A.D.Y.C. thanks the Croucher Foundation of Hong Kong for financial support. P.R.L.V.M.

gratefully acknowledges the support of a Human Capital and Mobility Fellowship from the European Union (Contract ERBCHBICT930781).

\section{REFERENCES}

[1] J. Wess and J. Bagger, Supersymmetry and Supergravity, 2nd. ed. (Princeton University Press, 1992).

[2] P.D. D'Eath and D.I. Hughes, Nucl. Phys. B 378, 381 (1992).

[3] J.Bene and R. Graham, gr-qc/9306017

[4] P.V. Moniz and J. Mourão, Class. Quantum Grav. 8, (1991) 1815 and references therein.

[5] E. Witten and J. Bagger, Phys. Lett. B 115 (1982).

[6] A.D.Y. Cheng, P.D. D'Eath and P.R.L.V. Moniz, DAMTP R94/13

[7] R. Capovilla and O. Obregon, Phys. Rev. D49 (1994) 6562.

[8] A.D.Y. Cheng, P.D. D’Eath and P.R.L.V. Moniz, Phys. Rev. D49 (1994) 5246. 